\title{
Gamma-ray and neutrino emission from misaligned microquasars
}

\author{
G. E. Romero ${ }^{1,2, \star}$ and M. Orellana $a^{1,2, \star \star}$ \\ 1 Instituto Argentino de Radioastronomía, CC5, (1894) Villa Elisa, Buenos Aires, Argentina \\ ${ }^{2}$ Facultad de Ciencias Astronómicas y Geofísicas, Universidad Nacional de La Plata, Paseo del Bosque, 1900 La Plata, \\ Argentina
}

Received 10 January 2005 / Accepted 22 April 2005

\begin{abstract}
Microquasars are accreting X-ray binary systems with non-thermal radio jets. In some of these systems the jet is expected to be strongly misaligned with the perpendicular to the orbital plane. If the donor star is an early-type star, the jet could collide with the stellar wind producing a standing shock between the compact object and the stellar surface. Relativistic particles injected by the jet can be re-accelerated and isotropized at the colliding region. If the jet has hadronic content, TeV protons will diffuse into the inner, dense wind leading to gamma-ray and neutrino production from interactions with the matter of the wind. In the case of very powerful jets, the wind pressure can be overbalanced and the jet might impact directly onto the stellar surface. We present estimates of the gamma-ray and neutrino luminosities for different sets of parameters in these scenarios and we briefly discuss the effects of this radiation on the donor star and its detectability with current instruments.
\end{abstract}

Key words. X-ray: binaries - gamma-rays: theory - gamma-rays: observations - neutrinos

\section{Introduction}

Microquasars (MQs) are accreting X-ray binary systems that have shown persistent or episodic non-thermal jet-like ejections (Mirabel \& Rodríguez 1999). It is believed that a significant fraction of all accreting black hole $(\mathrm{BH})$ and neutron star (NS) X-ray binaries are likely to exhibit MQ behavior at some epoch during their lifetime. Although it is not observationally required, the jet axis and the orbital plane of a given MQ system are usually assumed to be approximately perpendicular. However, Maccarone (2002) has shown that the timescale for the $\mathrm{BH}$ spin to align with the orbital angular momentum in binary systems is often longer than the lifetime of the system itself. Additionally, tidal effects upon the disk can induce a precession which could result in a severe misalignment (e.g. Larwood 1998; Kaufman et al. 2002). Precession and strong inclination of the jet can also be the result of the radiative warping of the inner accretion disk (e.g. Wijers \& Pringle 1999; Ogilvie \& Dubus 2001) or might be due to spin-spin interactions (e.g. Bardeen \& Petterson 1975; Sarazin et al. 1980). Observationally, there exists suggestive evidence for jet misalignments with the normal to the orbital plane in wind-fed accreting BHs. For instance, the jet axis lies at no more than $\sim 35$ degrees from the orbital plane in V4641 Sgr (see Butt et al. 2003, and references therein). Other systems like SS 433, LSI +61303 , and Cygnus X-3 are known to have precessing

\footnotetext{
* Member of CONICET, Argentina.

$\star \star$ Fellow of CONICET, Argentina,

e-mail: morellana@irma.iar.unlp.edu.ar
}

jets (see Katz 1980; Massi et al. 2004; and Mioduszewski et al. 2001, respectively). Precessing jets have been also suggested in the case of Cygnus X-1 (Romero et al. 2002).

In this paper we will consider the situation of a misaligned MQs with its jet lying close to the binary plane and being periodically directed straight to the companion star. Such a situation seems not to be unlikely. Butt et al. (2003), for instance, have considered the induced nucleosynthesis that should occur on the stellar surface as a consequence of the jet impact in such systems. Here, we will show that in the case of an earlytype companion star, the jet, under some conditions, could not reach the stellar surface since it is balanced by the strong stellar wind. However, another interesting feature appears: a strong shock is formed between the compact object and the star and protons injected by the jet can be re-accelerated and isotropized there. The most energetic of these protons then diffuse through the inner wind producing gamma-rays and neutrinos through $p+p \longrightarrow \pi^{0}+X$ and $p+p \longrightarrow \pi^{ \pm}+X$ reaction channels.

On the other hand, very powerful jets can overcome the pressure of the wind and directly impact on the star. In such a case the jet energy is dissipated through hadronic and electromagnetic cascades that develop in the stellar atmosphere. No continuum gamma-ray source above $\mathrm{MeV}$ energies is expected in this case, but a significant flux of high-energy neutrinos goes through the star. In what follows we characterize the general physical situation and estimate the gamma-ray and neutrino output of these systems, under a variety of conditions. The results will be of interest for current ground-based $\mathrm{TeV}$ gamma-ray telescopes and future satellites for $\mathrm{MeV}-\mathrm{GeV}$ 
gamma-ray astronomy, as well as for km-scale neutrino detectors.

\section{Jet-wind interaction}

In order to make specific calculations we will consider a misaligned MQ with a high-mass companion. The jet axis is assumed, for simplicity, to be parallel to the orbital radius $a$, and pointing toward the massive star of radius $R_{\star}$. As in Romero et al. (2003), we consider an expanding conical jet, i.e. the radius of the cross-section is $R_{\mathrm{j}}(d)=d \theta / 2$, where $\theta$ is the opening angle of the jet (in radians), $d$ is the distance from the compact object, and $r$ is the radial coordinate with origin at the center of the star $(d=a-r)$. Assuming adiabatic expansion, the magnetic field inside the conical jet changes with $d$ as

$B_{\mathrm{j}}(d)=B_{\mathrm{j}}\left(d_{0}\right)\left(\frac{d_{0}}{d}\right)$,

where $d_{0}$ is the distance from the jet's apex to the compact object. We will assume $d_{0}=50 R_{\mathrm{g}}$, being $R_{\mathrm{g}}$ the gravitational radius of the accreting star.

In order to determine the kinetic power of the jet we will adopt the jet-disk coupling hypothesis proposed by Falcke \& Biermann (1995) and applied with success to AGNs, i.e. the total jet power scales with the accretion rate as $L_{\mathrm{j}}=q_{\mathrm{j}} \dot{M}_{\mathrm{disk}} c^{2}$. We will assume that relativistic protons provide the dominant contribution to the jet power, i.e. $L_{\mathrm{j}} \approx \gamma_{\text {bulk }} \dot{N}_{\mathrm{p}} m_{\mathrm{p}} c^{2}$, where $\dot{N}_{\mathrm{p}}$ is the proton injection rate, $m_{\mathrm{p}}$ the proton mass, and $\gamma_{\text {bulk }}$ is the bulk Lorentz factor of the flow. The presence of relativistic hadrons has been inferred from iron X-ray line observations for the case of SS433 (e.g. Kotani et al. 1994, 1996; Migliari et al. 2002). Although direct and clear evidence exists only for this source so far, several models assume a hadronic content for the jets of some MQs (see Romero 2004, and references therein).

The high-mass companion star loses a significant fraction of its mass through a very strong supersonic wind, and then the mass transfer occurs mainly through this stellar wind. To ensure that there is no Roche lobe overflow the parameters of the binary system must satisfy the condition $R_{L}>R_{\star}$, where the Roche radius can be expressed in good approximation as

$R_{L} \approx a\left[0.38+0.2 \log \left(M_{\star} / M_{\mathrm{co}}\right)\right]$,

for $0.3 \leq\left(M_{\star} / M_{\mathrm{co}}\right) \leq 20$ (Kopal 1959). Here, $M_{\star}$ and $M_{\mathrm{co}}$ are the masses of the primary massive star and the compact object, respectively.

Typical mass loss rates and terminal wind velocities for early-type stars are of the order of $10^{-5} M_{\odot} \mathrm{yr}^{-1}$ and $2500 \mathrm{~km} \mathrm{~s}^{-1}$, respectively (Lamers \& Cassinelli 1999). The matter field will be determined by the continuity equation:

$\dot{M}_{\star}=4 \pi r^{2} \rho(r) v(r)$,

where $\rho$ is the density of the wind and $v(r)=v_{\infty}\left(1-R_{*} / r\right)^{\beta}$ is its velocity; $v_{\infty}$ is the terminal wind velocity, and the parameter $\beta$ is $\sim 1$ for very massive stars (Lamers \& Cassinelli 1999). Hence, assuming a gas dominated by protons, the particle density results:

$n(r)=\frac{\dot{M}_{\star}}{4 \pi m_{\mathrm{p}} r^{2} v(r)}$.
The pressure exerted by the stellar wind at a distance $r$ from the star is then:

$P_{\text {wind }}=\frac{\dot{M}_{\star} v(r)}{4 \pi r^{2}}$,

whereas the pressure due to the magnetic field of the star is

$P_{\text {mag }}=B(r)^{2} / 8 \pi$,

where $B(r)=\sqrt{B_{\phi}^{2}+B_{r}^{2}}$. The magnetic field of the star can be represented by the standard magnetic rotor model (Weber \& Davis 1967; White 1985; Lamers \& Cassinelli 1999): $B_{\phi} / B_{r}=\left(v_{\star} / v_{\infty}\right)\left(1+r / R_{\star}\right)$ and $B_{r}=B_{\star}\left(R_{\star} / r\right)^{2}$, where $v_{\star} \cong(0.1-0.2) v_{\infty}$ is the rotational velocity at the surface of the star (Conti \& Ebbets 1977), and $B_{\star}$ is the corresponding stellar magnetic field.

If the jet propagates with an opening angle $\theta$, at a distance $d$ from the compact object the ram pressure of the jet plasma is

$P_{\mathrm{j}}=\frac{L_{\mathrm{j}}}{\pi c \theta^{2} d^{2}}$.

As shown by Bednarek \& Protheroe (1997) for the case of AGN's jets, a strong stellar wind can stop the jet flow before it can reach the stellar surface. The interaction region will be formed by a contact discontinuity and two quasi-parallel shocks, one propagating backwards through the jet and the other moving toward the star through the wind.

The position $r_{\mathrm{s}}$ where the shock front will form can be obtained by equating the expression for the jet pressure to the expressions given in Eqs. (5) or (6). The location of the doubleshock structure will be determined by the specific parameters assumed for the jet and the stellar wind. As an example, we will work out the details of a family of models sharing the parameters listed in Table 1. For these values, it happens that $R_{L} \approx 1.53 R_{\star}$ and there is no Roche lobe overflow.

With this set of basic parameters, the ram pressure of the jet can not be balanced by the wind when $q_{\mathrm{j}}>0.006$. Above this value, however, the magnetic pressure can still balance and stop the jet. For $q_{\mathrm{j}}=10^{-2}$, a magnetic field of $B_{\star} \geq 200 \mathrm{G}$ is necessary to form a terminal shock front for the jet in the wind region. Early-type stars can support surface magnetic fields of even $\sim 10^{3} \mathrm{G}$ as reported by Donati et al. (2002). In order to study systems where the jet is balanced by the wind, we will assume different values for the stellar magnetic field and the jet power. After discussing these systems we will turn to MQs with very strong jets in Sect. 6 .

\section{Particle re-acceleration and diffusion}

The shocks generated by the interaction between the relativistic plasma in the jet and the stellar wind can accelerate particles by Fermi mechanism. For acceleration at a parallel shock we can write the acceleration rate as (e.g. Biermann \& Strittmatter 1987; Protheroe 1998):

$\left.\frac{\mathrm{d} E}{\mathrm{~d} t}\right|_{\mathrm{acc}}=\xi Z e c B$, with $\xi \simeq 0.015(u / c)^{2}$,

where $u$ is the shock velocity and all values are in cgs units. 
Table 1. Basic parameters assumed for the model.

\begin{tabular}{lll}
\hline \hline Parameter & Symbol & Value \\
\hline Mass of the compact object & $M_{\text {co }}$ & $10 M_{\odot}$ \\
Mass of the donor star & $\mathrm{M}_{\star}$ & $46.2 M_{\odot}$ \\
Jet's injection point & $d_{0}$ & $50 R_{\mathrm{g}}^{1}$ \\
Opening angle of the jet & $\theta$ & $5^{\circ}$ \\
Bulk Lorentz factor & $\gamma_{\text {bulk }}$ & 10 \\
Radius of the companion star & $R_{\star}$ & $15 R_{\odot}$ \\
Stellar effective temperature & $T_{\text {eff }}$ & $40000 \mathrm{~K}$ \\
Mass loss rate & $\dot{M}_{\star}$ & $10^{-5} M_{\odot} \mathrm{yr}^{-1}$ \\
Terminal wind velocity & $v_{\infty}$ & $2500 \mathrm{~km} \mathrm{~s}^{-1}$ \\
Compact object accretion rate & $\dot{M}_{\text {disk }}$ & $10^{-8} M_{\odot} \mathrm{yr}^{-1}$ \\
Wind velocity index & $\beta$ & 1 \\
Efficiency of the shock & $\chi$ & 0.1 \\
Orbital radius & $a$ & $3 R_{\star}$ \\
\hline
\end{tabular}

${ }^{1} R_{\mathrm{g}}=G M_{\mathrm{co}} / c^{2}$.

The highest energy gained by the particles in the process is imposed by the different energy losses (synchrotron emission, pp interactions and photopion production) or by the available space in the acceleration region. For the parameters considered in this paper the dominant energy loss is due to pp interactions with the stellar wind matter that pervades the acceleration region. For a relativistic proton $(E>1.22 \mathrm{GeV})$ this energy loss can be estimated as in Mannheim \& Schlickeiser (1994):

$-\left.\frac{\mathrm{d} E}{\mathrm{~d} t}\right|_{\mathrm{pp}} \approx 0.65 c n\left(r_{\mathrm{s}}\right) \sigma_{\mathrm{pp}}\left(E-m_{\mathrm{p}} c^{2}\right)$,

where $n\left(r_{\mathrm{s}}\right)$ is the particle density of the wind close to the shock front, and $\sigma_{\mathrm{pp}}$ is the inelastic cross section for pp interaction at the energy $E$. The maximum energy for the protons then results from

$$
\begin{aligned}
E_{\mathrm{p}}^{\max } \simeq & \min \left[1.9 \times 10^{15}\left(\frac{u}{c}\right)^{2} \frac{B_{\mathrm{j}}\left(d_{0}\right)}{\mathrm{G}} \mathrm{eV},\right. \\
& \left.3 \times 10^{14} \frac{B_{\mathrm{j}}\left(d_{0}\right)}{\mathrm{G}} \mathrm{eV}\right] .
\end{aligned}
$$

We shall adopt $u / c=0.1$ for the sub-relativistic shock and $u / c=0.5$ for a relativistic case. For the former $E_{\mathrm{p}}^{\max }$ is determined by the losses, whereas in the second case it is fixed by the size constrain, i.e. the maximum gyroradius for a proton is $\sim\left(a-R_{\star}\right)$.

The resulting proton spectrum from the diffusive acceleration will be a power-law extending from a minimum energy $\left(E_{\mathrm{p}}^{\min } \sim 10 \mathrm{GeV}^{1}\right)$ up to $E_{\mathrm{p}}^{\max }$ :

$N_{\mathrm{p}}(E)=K_{\Gamma} E^{-\Gamma}, 10 m_{\mathrm{p}} c^{2}<E<E_{\mathrm{p}}^{\max }$.

The index $\Gamma$ is $\sim 2$ for a strong non-relativistic shock and $\sim 1.5$ for a relativistic shock (e.g. Protheroe 1998).

Some fraction $\chi$ of the energy transported by the jet will be transferred to the particles accelerated at the shock front.

\footnotetext{
${ }^{1}$ From Table 1 the Lorentz factor of the jet is $\sim 10$ and then those protons at the lower energy tail of the co-moving particle energy distribution (for which the microscopic Lorentz factor is $\sim 1$ ) are injected at the shock with energies $\sim 10 \mathrm{GeV}$.
}

We will assume here an efficiency of $\chi=0.1$ (Blandford \& Ostriker 1978). Then, the constant $K_{\Gamma}$ in (11) can be obtained from

$\chi L_{\mathrm{j}}=\left(a-R_{\star}\right)^{2} c \int_{10 m_{\mathrm{p}} c^{2}}^{E_{\mathrm{p}}^{\max }} N_{\mathrm{p}}(E) E \mathrm{~d} E$.

The resulting population of relativistic protons will diffuse through the stellar wind, but not all of them will penetrate up to the densest parts where the probability of pp interactions is higher because of the modulation effect of the wind (Romero $\&$ Torres 2003; Torres et al. 2004). Whether the particles can reach the base of the wind or not is given by the ratio $(\epsilon)$ of the diffusion and convection timescales, which are given by $t_{\mathrm{d}}=3 r^{2} / D$ and $t_{\mathrm{c}}=3 r / v(r)$, respectively. Here $D$ is the diffusion coefficient defined as (Ginzburg \& Syrovatskii 1964):

$D \sim \frac{1}{3} \lambda_{r} c$

where $\lambda_{r}$ is the mean-free-path for diffusion in the radial direction. Only particles for which $\epsilon<1$ will be able to overcome convection and enter the dense wind region to produce $\gamma$-rays through pp interactions.

The value of the diffusion coefficient is of course not wellknown, especially for such a complex and turbulent medium as the wind of a massive star. Following White (1985) we will make use of the approximation given by Völk \& Forman (1982), assuming that the mean-free-path for scattering parallel to the magnetic field direction is $\lambda_{\|} \sim 10 r_{\mathrm{g}}=10 \mathrm{E} / \mathrm{eB}$, where $r_{\mathrm{g}}$ is the proton gyro-radius and $E$ the proton energy. The value of $\lambda$ in the perpendicular direction is shorter: $\lambda_{\perp} \sim r_{\mathrm{g}}$. The meanfree-path in the radial direction is then (see Torres et al. 2004):

$\lambda_{r}=r_{\mathrm{g}}\left(10 \cos ^{2} \theta+\sin ^{2} \theta\right)$,

where

$\cos ^{-2} \theta=1+\left(B_{\phi} / B_{r}\right)^{2}$.

Through $\epsilon=1$ we find now the minimum energy $E_{\mathrm{p}}^{\min }(r)$ below which the particles are taken away by convection in the wind (see the explicit expression for $\epsilon$ in the paper by Torres et al. 2004). In what follows, we will take $E_{\mathrm{p}}^{\min }\left(r_{\mathrm{s}}\right)$ as the minimum energy of a proton accelerated at the shock front to collide with a proton of the inner wind.

\section{Gamma-ray production}

We can now proceed to calculate the $\pi^{0}$ gamma-ray luminosity from the inner wind. The differential $\gamma$-ray emissivity from $\pi^{0}$-decays can be approximated by

$q_{\gamma}\left(E_{\gamma}\right)=4 \pi \sigma_{\mathrm{pp}}\left(E_{\mathrm{p}}\right) \frac{2 Z_{\mathrm{p} \rightarrow \pi^{0}}^{(\Gamma)}}{\Gamma} J_{\mathrm{p}}\left(E_{\gamma}\right) \eta_{\mathrm{A}} \Theta\left(E_{\mathrm{p}}-E_{\mathrm{p}}^{\mathrm{min}}\right)$,

at the energies of interest here. $Z_{\mathrm{p} \rightarrow \pi^{0}}^{(\Gamma)}$ is the so-called spectrum weighted moment, defined as:

$Z_{\mathrm{p} \rightarrow \pi^{0}}^{(\Gamma)}=\int_{0}^{1} x^{\Gamma-1} F_{\mathrm{p} \pi^{0}}(x) \mathrm{d} x$, 
Table 2. Some results about the high-energy photon emission in misaligned MQs. The $A, B, C$ letters naming the model indicate a high, middle or low jet-disk coupling, respectively.

\begin{tabular}{cccccccccc}
\hline \hline Model & $q_{\mathrm{j}}$ & $\begin{array}{c}B_{\star} \\
{[\mathrm{G}]}\end{array}$ & $\begin{array}{c}B_{\mathrm{j}}\left(d_{0}\right) \\
{[\mathrm{G}]}\end{array}$ & $\begin{array}{c}r_{\mathrm{s}} \\
{\left[R_{\odot}\right]}\end{array}$ & $\begin{array}{c}E_{\mathrm{p}}^{\min } \\
{[\mathrm{TeV}]}\end{array}$ & $\begin{array}{c}E_{\mathrm{p}, \mathrm{sub}}^{\max }[\mathrm{TeV}] \\
{[\mathrm{TeV}]}\end{array}$ & $\begin{array}{c}E_{\mathrm{p}, \mathrm{rel}}^{\max } \\
{[\mathrm{TeV}]}\end{array}$ & $\begin{array}{c}L_{\gamma,(\Gamma=2)} \\
{\left[\mathrm{erg} \mathrm{s}^{-1}\right]}\end{array}$ & $\begin{array}{c}L_{\gamma,(\Gamma=1.5)} \\
{\left[\mathrm{erg} \mathrm{s}^{-1}\right]}\end{array}$ \\
\hline$A 1$ & $10^{-2}$ & 500 & 10 & 21.7 & 92.2 & 190 & 3000 & $2.2 \times 10^{32}$ & $4.1 \times 10^{32}$ \\
$A 2$ & $10^{-2}$ & 500 & 100 & 21.7 & 92.2 & 1900 & 30000 & $7.5 \times 10^{32}$ & $3.9 \times 10^{32}$ \\
$B 1$ & $10^{-3}$ & 1 & 10 & 34.2 & 0.2 & 190 & 3000 & $1.9 \times 10^{32}$ & $5.4 \times 10^{31}$ \\
$B 2$ & $10^{-3}$ & 1 & 100 & 34.2 & 0.2 & 1900 & 30000 & $2.1 \times 10^{32}$ & $4.3 \times 10^{31}$ \\
$B 3$ & $10^{-3}$ & 100 & 10 & 34.2 & 21.3 & 190 & 3000 & $6.4 \times 10^{31}$ & $4.7 \times 10^{31}$ \\
$B 4$ & $10^{-3}$ & 100 & 100 & 34.2 & 21.3 & 1900 & 30000 & $1.1 \times 10^{32}$ & $4.1 \times 10^{31}$ \\
$C 1$ & $10^{-4}$ & 1 & 10 & 41.1 & 0.2 & 190 & 3000 & $1.9 \times 10^{31}$ & $5.4 \times 10^{30}$ \\
$C 2$ & $10^{-4}$ & 1 & 100 & 41.1 & 0.2 & 1900 & 30000 & $2.1 \times 10^{31}$ & $4.3 \times 10^{30}$ \\
$C 3$ & $10^{-4}$ & 100 & 10 & 41.1 & 20.8 & 190 & 3000 & $6.5 \times 10^{30}$ & $4.7 \times 10^{30}$ \\
$C 4$ & $10^{-4}$ & 100 & 100 & 41.1 & 20.8 & 1900 & 30000 & $1.1 \times 10^{31}$ & $4.1 \times 10^{30}$ \\
\hline
\end{tabular}

where $x=E_{\pi^{0}} / E_{\mathrm{p}}$ and $F_{\mathrm{p} \pi^{0}}(x)$ is the dimensionless inclusive cross section (Gaisser 1990, p. 31). The parameter $\eta_{\mathrm{A}}$ reflects the contribution from different nuclei in the wind (for the standard composition of an early-type star we will assume $\left.\eta_{\mathrm{A}} \sim 1.5\right) . J_{\mathrm{p}}\left(E_{\gamma}\right)=(c / 4 \pi) N_{\mathrm{p}}\left(E_{\gamma}\right)$ is the proton flux distribution evaluated at $E=E_{\gamma}$ (units of protons per unit time, solid angle, energy-band, and area).

The inelastic cross section for pp interactions at energy $E_{\mathrm{p}} \approx 6 \xi_{\pi^{0}} E_{\gamma} / K$, where $K \sim 0.5$ is the inelasticity coefficient and $\xi_{\pi^{0}}=1.1\left(E_{\mathrm{p}} / \mathrm{GeV}\right)^{1 / 4}$ is the multiplicity for the neutral pions (e.g. Ginzburg \& Syrovatskii 1964), can be represented above $E_{\mathrm{p}} \approx 10 \mathrm{GeV}$ by the expression given in Aharonian $\&$ Atoyan (1996). Typically, at $1 \mathrm{TeV}, \sigma_{\mathrm{pp}} \sim 34 \mathrm{mb}$. Finally, $\Theta\left(E_{\mathrm{p}}-E_{\mathrm{p}}^{\mathrm{min}}\right)$ is a Heaviside function that takes into account the fact that only protons with energies higher than $E_{\mathrm{p}}^{\min }\left(r_{\mathrm{s}}\right)$ will diffuse into the densest part of the wind. The spectral $\gamma$-ray intensity (photons per unit time and energy-band) is:

$I_{\gamma}\left(E_{\gamma}\right)=\int n(r) q_{\gamma}\left(E_{\gamma}\right) \mathrm{d} V$

where $V$ is the interaction volume. The generated luminosity in a given band $\left(E_{2}, E_{1}\right)$ then is

$L_{\gamma}=\int_{E_{1}}^{E_{2}} E_{\gamma} I_{\gamma}\left(E_{\gamma}\right) \mathrm{d} E_{\gamma}$

(see, e.g., Romero et al. 2003, for details).

In order to estimate the $\gamma$-ray luminosity that could be produced in the jet-wind interaction of a misaligned MQ with a high-mass companion, we have fixed the parameters that describe the geometry of this scenario for a standard case (Table 1). Note that the radius of the star holds a $\sim 26.5$ angle as seen from the compact object, so the star fills $\sim 10.5 \%$ of the compact object's sky. We have calculated the luminosity in high-energy photons for different jet-disk coupling values $\left(q_{\mathrm{j}}\right)$, and for different values of $B_{\star}$ and $B_{\mathrm{j}}\left(d_{0}\right)$. The most effective particle acceleration occurs at the relativistic plasma. We have considered a sub-relativistic strong shock that leads to a spectrum with $\Gamma \sim 2$ and a mildly relativistic shock with $u=0.5 c$ $(\Gamma \sim 1.5)$. Table 2 characterizes (in the first three columns) a number of specific models, ordered by decreasing jet power, and then gives the location of the shock front, the maximum and minimum energy for protons penetrating into the base of the wind, and the integrated gamma-ray luminosities generated for the sub-relativistic and the relativistic cases of particle acceleration.

The spectral high-energy distributions for some of the computed models are shown in Fig. 1. The plots correspond to $L_{\gamma}\left(E_{\gamma}\right)=E_{\gamma}^{2} I_{\gamma}\left(E_{\gamma}\right)$, with an exponential-type cutoff at $E_{\gamma} \sim$ $\left(E_{\mathrm{p}}^{\max } / \mathrm{GeV}\right)^{3 / 4} / 20$. The obtained isotropic luminosities above $1 \mathrm{TeV}$ are in the range $10^{30}-10^{32} \mathrm{erg} \mathrm{s}^{-1}$.

High-energy gamma rays can be absorbed in the stellar photon field through pair production, and then inverse Compton emission from these pairs can initiate an $e^{ \pm}$-pair cascade. This effect has been studied in detail by Bednarek (1997, 2000) and more recently by Sierpowska \& Bednarek (2005). Figure 2 shows the $\tau_{\gamma}$ opacity of the stellar photosphere to gamma-ray propagation as a function of the gamma-photon energy and the distance $r$ from the star where it is created. This opacity, for a photon moving radially from the massive star, can be roughly computed, averaging over the injection angles, as:

$\tau_{\gamma}\left(E_{\gamma}, r\right)=\int_{r}^{\infty} \int_{0}^{\infty} N\left(E_{\star}, r^{\prime}\right) \sigma_{\mathrm{e}^{-} \mathrm{e}^{+}}\left(E_{\star}, E_{\gamma}\right) \mathrm{d} E_{\star} \mathrm{d} r^{\prime} ，$

where $E_{\star}$ is the energy of the photons emitted by the star, $E_{\gamma}$ is the energy of the $\gamma$-ray, and $\sigma_{\mathrm{e}^{-} \mathrm{e}^{+}}\left(E_{\star}, E_{\gamma}\right)$ is the cross section for photon-photon pair production (e.g. Lang 1980). The stellar photon distribution is that of a blackbody peaking at the star's effective temperature $\left(T_{\text {eff }}\right)$ :

$N\left(E_{\star}, r\right)=\left(\pi B\left(E_{\star}\right) / h E_{\star} c\right) R_{\star}^{2} / r^{2}$,

where $h$ is the Planck constant and

$B\left(E_{\star}\right)=\left[2 E_{\star}^{3} /(h c)^{2}\right] /\left(\mathrm{e}^{E_{\star} / k T_{\text {eff }}}-1\right)$.

The inverse Compton opacity $\tau_{\mathrm{IC}}\left(E_{\mathrm{e}}, r\right)$ determines the advance of the cascades after the pair creation. We have estimated that gamma-rays with energies below $\sim 10^{12} \mathrm{eV}$ will be mostly absorbed, triggering IC cascades. More energetic photons $(>10 \mathrm{TeV})$ will escape. The result will be a soft spectrum at $\mathrm{GeV}$ energies (Bednarek \& Protheroe 1997) and a peak at high energies (Fig. 1). 

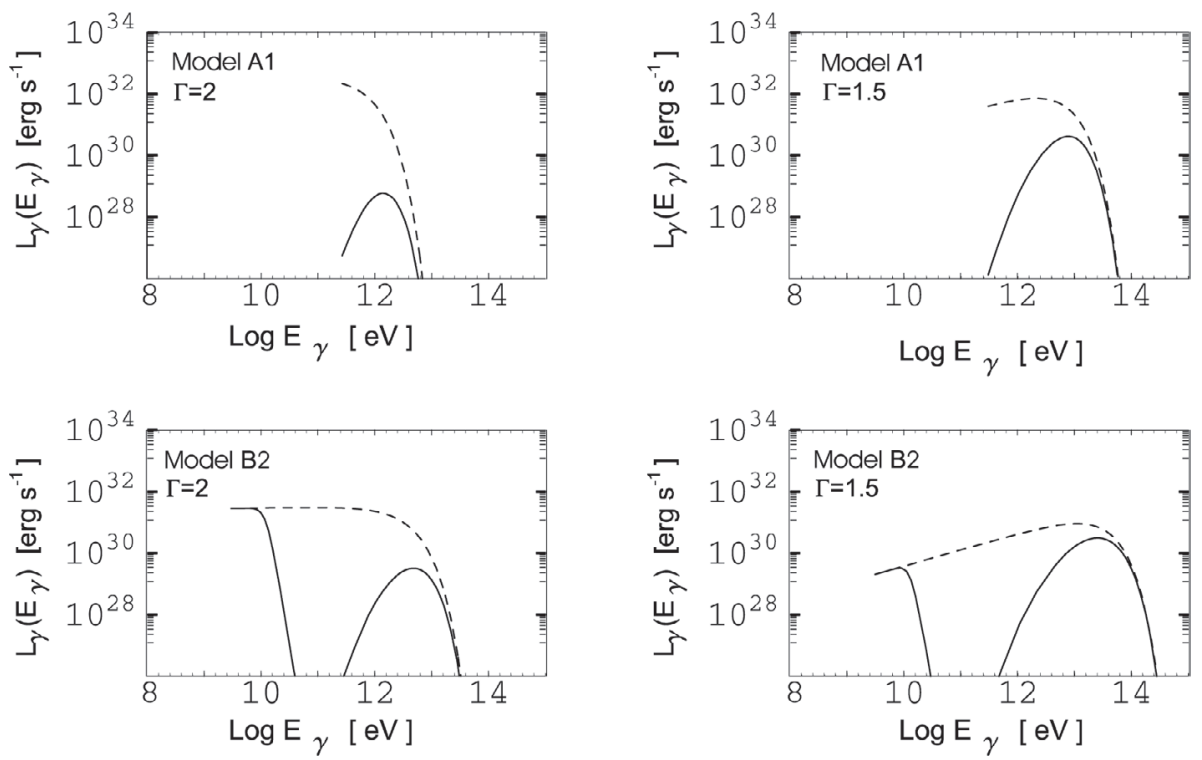

Fig. 1. Spectral high-energy distribution at $\mathrm{TeV}$ energies from the jet-wind interaction in a misaligned MQ. The left panel corresponds to $\Gamma=2$, and the right to $\Gamma=1.5$ (see text). The dashed lines correspond to the production spectra whereas the solid lines represent the absorbed spectra.
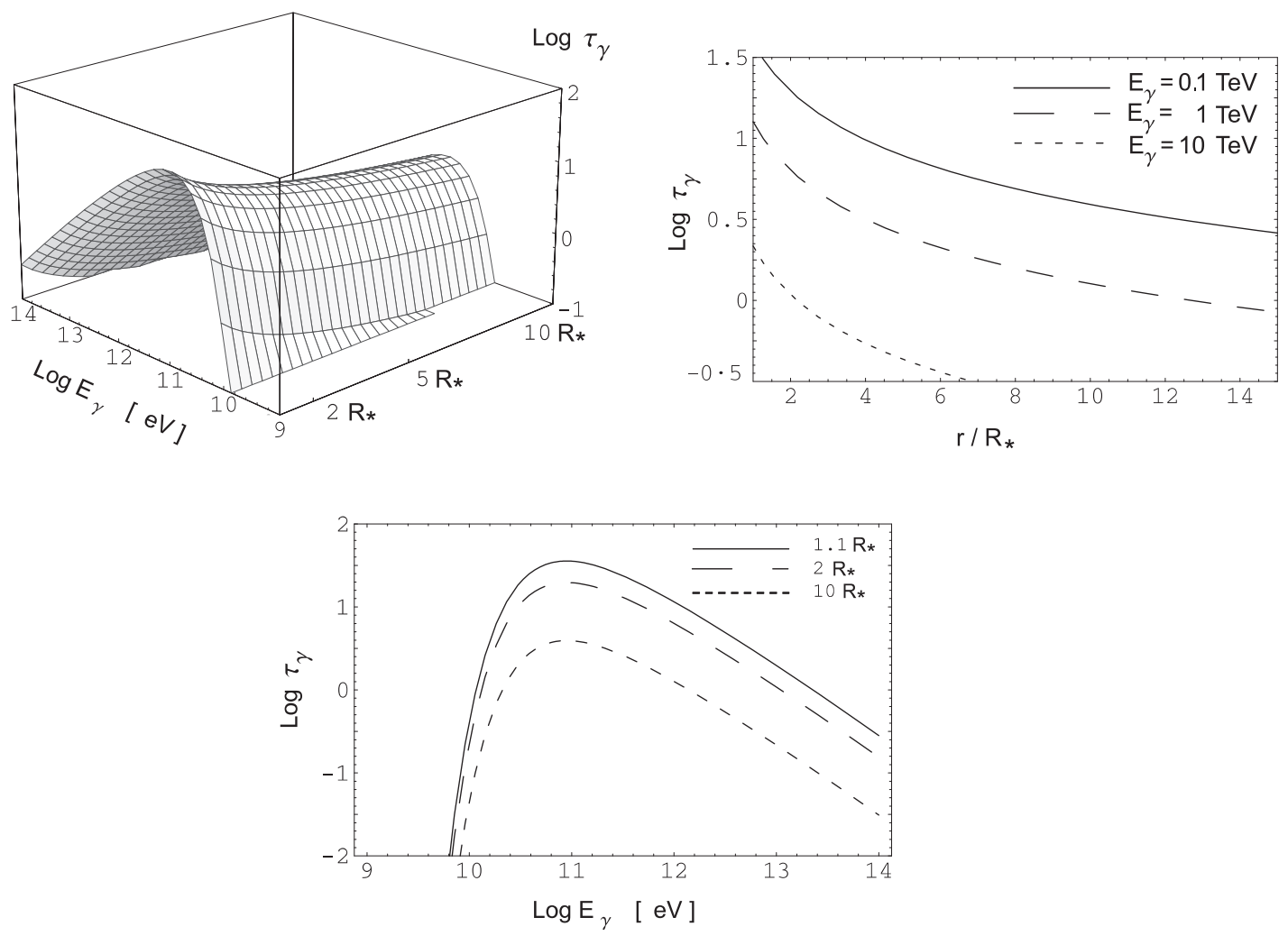

Fig. 2. Stellar photosphere's opacity due to photo-pair creation, as function of the $\gamma$-ray energy and the distance from the star, for a photon moving radially from the massive star. Upper-right: optical depth for fixed values of energy. Lower panel: optical depth for fixed distances.

\section{Neutrino production and possible detection}

The $v_{\mu}+\bar{v}_{\mu}$ neutrino intensity $I_{v}\left(E_{v}\right)$ may be derived from the spectral $\gamma$-ray intensity by imposing energy conservation (Alvarez-Muñiz \& Halzen 2002; Torres et al. 2004):

$\int E_{\gamma} I_{\gamma}\left(E_{\gamma}\right) \mathrm{d} E_{\gamma}=C \int E_{v} I_{\gamma}\left(E_{v}\right) \mathrm{d} E_{v}$ where the limits of the integrals are $E_{\gamma[v]}^{\min }\left(E_{\gamma[v]}^{\max }\right)$, the minimum (maximum) energy of the photons (neutrinos) and $C$ is a numerical constant of order one (i.e. the total neutrino luminosity produced by the decay of charged pions is a comparable quantity with that of the photons $L_{\gamma}$ ). The neutrino intensity will be given by

$I_{v}\left(E_{v}\right)=C_{v}\left(E_{v} / \mathrm{erg}\right)^{-\Gamma} \mathrm{e}^{-E_{v} / E_{v}^{\max }}$ 
Table 3. Some results about the neutrino emission in misaligned MQs. Case $D 1$ corresponds to the direct impact of a jet with $q_{\mathrm{j}}=0.1$ onto the stellar surface.

\begin{tabular}{ccc}
\hline \hline Model & $\begin{array}{c}C_{v,(\mathrm{\Gamma}=2)} \\
\mathrm{erg}^{-1} \mathrm{~s}^{-1}\end{array}$ & $\begin{array}{c}C_{v,(\mathrm{\Gamma}=1.5)} \\
\mathrm{erg}^{-1} \mathrm{~s}^{-1}\end{array}$ \\
\hline$A 1$ & $4.0 \times 10^{32}$ & $1.7 \times 10^{32}$ \\
$A 2$ & $3.3 \times 10^{32}$ & $5.4 \times 10^{31}$ \\
$B 1$ & $3.7 \times 10^{31}$ & $1.6 \times 10^{31}$ \\
$B 2$ & $3.1 \times 10^{31}$ & $5.4 \times 10^{30}$ \\
$B 3$ & $3.9 \times 10^{31}$ & $1.7 \times 10^{31}$ \\
$B 4$ & $3.3 \times 10^{31}$ & $5.4 \times 10^{30}$ \\
$C 1$ & $3.7 \times 10^{30}$ & $1.6 \times 10^{30}$ \\
$C 2$ & $3.1 \times 10^{30}$ & $5.4 \times 10^{29}$ \\
$C 3$ & $3.9 \times 10^{30}$ & $1.7 \times 10^{30}$ \\
$C 4$ & $3.3 \times 10^{30}$ & $5.5 \times 10^{29}$ \\
\hline$D 1$ & $3.7 \times 10^{32}$ & $3.1 \times 10^{34}$ \\
\hline
\end{tabular}

where the constant $C_{v}$ is in $\mathrm{erg}^{-1} \mathrm{~s}^{-1}$. The values of $C_{v,(\Gamma=1.5)}$ and $C_{v,(\Gamma=2)}$ for the different models are listed in Table 3.

The neutrinos are produced in the inner wind around the star. A fraction of them, which will depend on the specific geometry of the system, will be intercepted by the star. The star, in turn, can absorb some of these neutrinos. We have estimated the neutrino energy deposition rate $\eta_{v}$ (see Gaiser et al. 1986) inside the star. The results confirm that no effects of neutrino heating will be observable during the stellar lifetime. This fact is expected since only a small fraction of the power of the jet is directed toward the star by neutrinos $\left(\eta_{v} \sim 10^{27}-10^{30} \mathrm{erg} \mathrm{s}^{-1}\right)$ and this represents a very small fraction $\left(10^{-11}-10^{-8}\right)$ of the luminosity of the star.

Although the neutrino emission has no effect upon the companion star, perhaps it could be detectable at the Earth. If the distance to the system is $d_{\mathrm{MQ}}$, the neutrino flux arriving to a km-scale detector like ICECUBE will be $F_{v}\left(E_{v}\right)=$ $I_{v}\left(E_{v}\right) /\left(4 \pi d_{\mathrm{MQ}}^{2}\right)$. To make some quantitative estimate we adopt in what follows $d_{\mathrm{MQ}} \sim 5 \mathrm{kpc}$.

The neutrino signal can be estimated as (Anchordoqui et al. 2003)

$S=T_{\mathrm{obs}} \int \mathrm{d} E_{v} A_{\mathrm{eff}} F_{v}\left(E_{v}\right) P_{v \rightarrow \mu}\left(E_{v}\right)$,

where $P_{v \rightarrow \mu}\left(E_{v}\right) \approx 1.3 \times 10^{-6}\left(E_{v} / \mathrm{TeV}\right)^{0.8}$ denotes the probability that a $v$ of energy $E_{v} \sim 1-10^{3} \mathrm{TeV}$, on a trajectory through the detector, produces a muon (Gaisser et al. 1995). $T_{\text {obs }}$ is the observing time and $A_{\text {eff }}$ the effective area of the detector. On the other hand, the noise will be given by

$N=\left[T_{\text {obs }} \int \mathrm{d} E_{v} A_{\text {eff }} F_{\mathrm{B}}\left(E_{v}\right) P_{\nu \rightarrow \mu}\left(E_{v}\right) \Delta \Omega\right]^{1 / 2}$,

where $\Delta \Omega$ is the solid angle of the search bin $\left(\Delta \Omega_{1^{\circ} \times 1^{\circ}} \approx 3 \times\right.$ $10^{-4} \mathrm{sr}$ for an instrument like ICECUBE, Karle 2002) and

$F_{\mathrm{B}}\left(E_{v}\right) \leq 0.2\left(E_{v} / \mathrm{GeV}\right)^{-3.21} \mathrm{GeV}^{-1} \mathrm{~cm}^{-2} \mathrm{~s}^{-1} \mathrm{sr}^{-1}$

is the $v_{\mu}+\bar{v}_{\mu}$ atmospheric $v$-flux (Volkova 1980; Lipari 1993). Using as the integration limits the minimum and maximum energies detectable by the instrument (i.e. $1-10^{5} \mathrm{TeV}$ ) we obtain $T_{\text {obs }}$ for a signal-to-noise ratio $S / N=3$. The results are in all the cases (models A1 to $\mathrm{C} 4$ ) greater than several thousands years, rendering the detection impractical with the current sensitivity. Once again, these are lower limits, since the duty cycle is not 1 . Only in the case of very powerful jets with $q_{\mathrm{j}}>0.1 \mathrm{a}$ detection might be possible on human-life timescales. We will discuss this case in the next section.

\section{Powerful jets and direct interaction with the star}

We consider now a heavy jet directly impacting the $\operatorname{star}\left(q_{\mathrm{j}}=\right.$ $\left.0.1^{2}\right)$. The proton spectrum of the relativistic jet flow is assumed to be a power law $N_{\mathrm{p}}^{\prime}\left(E_{\mathrm{p}}^{\prime}\right)=K_{\Gamma} E_{\mathrm{p}}^{\prime-\Gamma}$, valid for $E_{\mathrm{p}}^{\prime \text { min }} \leq$ $E_{\mathrm{p}}^{\prime} \leq E_{\mathrm{p}}^{\prime \max }$, in the co-moving jet frame. The corresponding particle flux will be $J_{\mathrm{p}}^{\prime}\left(E_{\mathrm{p}}^{\prime}\right)=(c / 4 \pi) N_{\mathrm{p}}^{\prime}\left(E_{\mathrm{p}}^{\prime}\right)$. Since the jet expands (in a conical way), the proton flux can be written as:

$J_{\mathrm{p}}^{\prime}\left(E_{\mathrm{p}}^{\prime}\right)=\frac{c}{4 \pi} K_{\Gamma}\left(\frac{d_{0}}{d}\right)^{n} E_{\mathrm{p}}^{\prime-\Gamma}$,

where $n$ is positive and we assume $n=2$ (which corresponds to the conservation of the number of particles, see Ghisellini et al. 1985), and a prime refers to the jet frame. Using relativistic invariants, it can be proved that the proton flux reaching the surface of the star, in the lab frame, becomes (e.g. Romero et al. 2003)

$$
\begin{aligned}
J_{\mathrm{p}}\left(E_{\mathrm{p}}\right)= & \frac{c K_{\Gamma}}{4 \pi}\left(\frac{d_{0}}{a-R_{\star}}\right)^{n} \\
& \times \frac{\gamma_{\mathrm{bulk}}^{-\Gamma}\left(E_{\mathrm{p}}+\beta_{\mathrm{b}} \sqrt{E_{\mathrm{p}}^{2}-m_{\mathrm{p}}^{2} c^{4}}\right)^{-\Gamma}}{\left(1+\frac{\beta_{\mathrm{b}} E_{\mathrm{p}}}{\sqrt{E_{\mathrm{p}}^{2}-m_{\mathrm{p}}^{2} c^{4}}}\right)},
\end{aligned}
$$

where $\gamma_{\text {bulk }}$ is the jet Lorentz factor, $\beta_{\mathrm{b}}$ is the corresponding velocity in units of $c$, and we have considered protons with small transverse momentum (hence the lack of dependency in the particle angle when compared with the corresponding expression in Romero et al. 2003).

The number density $n_{0}{ }^{\prime}$ of particles flowing in the jet at $R_{0}=R\left(d_{0}\right)$ can be determined as in Romero et al. (2003) and the normalization constant $K_{\Gamma}$ can be derived provided that $E_{\mathrm{p}}^{\prime \max } \gg{E_{\mathrm{p}}^{\prime m i n}}^{\prime \min }$. Since $E_{\mathrm{p}}^{\prime \min } \sim 1 \mathrm{GeV}$, this is always the case, and

$K_{\Gamma}=n_{0}{ }^{\prime}(\Gamma-1)\left(E_{\mathrm{p}}^{\prime \min }\right)^{\Gamma-1}$.

In the numerical calculations we have considered $E_{\mathrm{p}}^{\prime \max }=$ $100 \mathrm{TeV}, \gamma_{\text {bulk }}=10$, and two cases for the proton energy distribution in the jet ${ }^{3}: \Gamma=2$ and $\Gamma=1.5$.

The gamma-rays generated by the jet-star interaction will be absorbed by the electromagnetic cascades in the star. The main signature of such cascades could be a rather broad $\mathrm{e}^{+}-\mathrm{e}^{-}$ annihilation line. The neutrino flux from charged pion decays

\footnotetext{
${ }^{2} L_{\mathrm{j}}=q_{\mathrm{j}} 5.7 \times 10^{38} \mathrm{erg} \mathrm{s}^{-1}$.

${ }^{3}$ Notice that these are the original particle distributions in the jet and not the result of re-acceleration at the colliding shock region as before. Here the jet impacts directly in a high-density region.
} 
will be significantly larger than in the case of the jet-wind interactions. For the density profile of the star we have used

$\rho(r)=\rho_{\mathrm{c}}\left[1-\left(r / R_{\star}\right)^{2}\right]$,

with $\rho_{\mathrm{c}} \simeq 0.05 \mathrm{~g} / \mathrm{cm}^{3}$, in accordance with the adopted stellar mass.

In the lab frame, the neutrino energies reach $\sim 1 \mathrm{TeV}$, then only the hard energy tail of the neutrino spectrum lies in the range of observation of ICECUBE. The neutrino energy deposition rate for $\Gamma=1.5$ results $\eta_{v} \simeq 2.4 \times 10^{33} \mathrm{erg} \mathrm{s}^{-1}$. The neutrino signal on the Earth produces a number of muons per year of $N_{\mu}=3.25$. This means a detectable signal at $3 \sigma$-level for an observing time $T_{\mathrm{obs}} \sim 15$ years, in the case of a source at $2 \mathrm{kpc}$ and a close alignment of the jet with the line of sight and a duty cycle of $20 \%$.

\section{Comments}

The type of misaligned MQ discussed in Sect. 4 is a variable gamma-ray source. For the masses and orbital radius given in Table 1, the orbital period is $\sim 2.5$ days. Since the star occupies $\sim 10 \%$ of the sky of the compact object and there are two jets in the orbital plane, the expected duty cycle is $\sim 20 \%$. The gamma-ray emission is ignited twice per orbit, every $\sim 30 \mathrm{~h}$. The source remains active for around $\sim 6 \mathrm{~h}$ each time, since the diffusion timescale for the accelerated particles is much shorter than this value: $t_{\mathrm{d}}\left(E_{\mathrm{p}}^{\max }\right) \lesssim 29 \mathrm{~s}$ and $t_{\mathrm{d}}\left(E_{\mathrm{p}}^{\min }\right) \lesssim 203 \mathrm{~s}$ for the models considered here. All these numbers will change, of course, from one case to another depending on the specific configuration, but in all cases the result will be a variable source unless the diffusion timescale is much longer than the interval between the different jet-wind collisions.

Because of the isotropization of the particle flux at the shock front located at $r_{\mathrm{s}}$ the gamma-ray emission will not be as much dependent on the viewing angle as in the case of pure relativistic jet models (e.g. Romero et al. 2003). Occultation events will occur only if the observer lies close to the orbital plane. Such events would be rare since they require an alignment among compact object, star and observer. The flux, additionally, would not be completely suppressed since the relativistic particles diffuse around the entire inner wind.

Since misaligned MQs can be $\mathrm{TeV}$ and (through electromagnetic cascades in the stellar photosphere) $\mathrm{MeV}-\mathrm{GeV}$ sources, we could ask whether some of the unidentified gamma-ray sources detected by EGRET instrument might be associated with them. There are $\sim 20$ unidentified variable sources at low galactic latitudes in the Third EGRET Catalog (Hartman et al. 1999; Torres et al. 2001; Nolan et al. 2003). Bosch-Ramon et al. (2004) have shown that they form a distinct population with a distribution consistent with what is expected for high-mass MQs. The total number of high-mass X-ray binaries brighter than $10^{34} \mathrm{erg} \mathrm{s}^{-1}$ at X-rays in the Galaxy is estimated in $\sim 380$ (Grimm et al. 2002). Only a small fraction of them will have the type of persistent jets necessary to produce detectable gamma-ray sources. Around 20 sources seems to be a reasonable estimate (Bosch-Ramon et al. 2004). If the original orientation angles of the jets are randomly distributed we could expect that in 10-20\% of these systems the jet could be periodically directed towards the companion star (Butt et al. 2003). This means that 2-4 of the variable EGRET sources at low galactic latitudes might be associated with misaligned MQs. Hints of periodic variability could help to identify these sources. Their main footprints, however, would be a stronger $\mathrm{TeV}$ emission than what is expected in normal MQs. Hence, future observations with Cherenkov telescopes like HESS, MAGIC or Veritas might lead to the identification of these sources.

\section{Conclusions}

The jets of MQs can form a small angle respect to the orbital plane. In some high-mass X-ray binary systems the jet could be directed straight to the companion star. We have shown that in such a situation the wind of the star can balance the jet pressure in many cases. A jet-wind collision region is formed between the star and the compact object, with a contact discontinuity separating both shocked media. Relativistic particles injected by the jet in the strong shock can be re-accelerated up to very high energies and isotropized in this region. These particles diffuse into the inner wind as far as diffusion dominates over convection. Gamma-rays and neutrinos can be then produced in the densest part of the wind. We have presented calculations of the gamma-ray luminosity, which might be detectable through high-energy telescopes. The neutrino flux is usually below the sensitivity of $\mathrm{km}$-scale detectors like ICECUBE unless extremely powerful jets are assumed. Such jets could reach the star, triggering cascades and producing nucleosynthesis there, as discussed by Butt et al. (2003). We have also shown that photon-photon absorption of gamma-rays with energies below $10 \mathrm{TeV}$ can initiate electromagnetic cascades in the stellar photosphere, leading to $\mathrm{MeV}-\mathrm{GeV}$ sources that might contribute to the population of variable gamma-ray sources detected by EGRET at low galactic latitudes. Future instruments like GLAST will help to unveil the nature of these sources.

Acknowledgements. We thank J. M. Paredes and V. Bosch-Ramon for valuable discussions and comments on the manuscript. We also thank an anonymous referee who made important suggestions that led to a significant improvement of the manuscript. This research has been supported by Fundación Antorchas, CONICET, and the Argentine agency ANPCyT through Grant PICT 03-13291.

\section{References}

Aharonian, F. A., \& Atoyan, A. M. 1996, A\&A, 309, 917

Anchordoqui, L. A., Torres, D. F., McCauley, T., et al. 2003, ApJ, 589, 481

Alvarez-Muñiz, J., \& Halzen, F. 2002, ApJ, 576, L33

Bardeen, J. M., \& Petterson, J. A. 1975, ApJ, 195, L65

Bednarek, W. 1997, A\&A, 322, 523

Bednarek, W. 2000, A\&A, 362, 646

Bednarek, W., \& Protheroe, R. J. 1997, MNRAS, 287, L9

Biermann, P. L., \& Strittmatter, P. A. 1987, ApJ, 322, 643

Blandford, R. D., \& Ostriker J. P. 1978, ApJ, 221, L29

Bosch-Ramon, V., Romero, G. E., \& Paredes, J. M. 2005, A\&A, 429, 267

Butt, M. Y., Maccarone, J. T., \& Prantzos, N. 2003, ApJ, 587, 748

Conti, P. S., \& Ebbets, D. 1977, ApJ, 213, 438 
Donati, J. F., Babel, J., Harries, T. J., et al. 2002, MNRAS, 333, 55

Falcke, H., \& Biermann, P. L. 1995, A\&A, 293, 665

Gaisser, T. K. 1990, Cosmic Rays and Particle Physics (Cambridge: Cambridge University Press)

Gaisser, T. K., Stecker, F. W., Harding, A. K., \& Barnard, J. J. 1986, ApJ, 309, 674

Gaisser, T. K., Halzen, F., \& Stanev, T. 1995, Phys. Rep., 258, 173 [Erratum-ibid., 271, 355 (1996)]

Ghisellini, G., Maraschi, L., \& Treves, A. 1985, A\&A, 146, 204

Ginzburg, V. L., \& Syrovatskii S. I. 1964, The Origin of Cosmic Rays (New York: Pergamon Press)

Grimm, H.-J., Gilfanov, M., \& Sunyaev, R. 2002, A\&A, 391, 923

Hartman, R. C., Bertsch, D. L., \& Bloom, S. D., et al. 1999, ApJS, 123,79

Katz, J. I. 1980, ApJ, 236, L127

Kaufman Bernadó, M. M., Romero, G. E., \& Mirabel, I. F. 2002, A\&A, 385, L10

Kopal, Z. 1959, Close binary systems (New York: J. Wiley \& Sons)

Kotani, T., Kawai, N., Aoki, T., et al. 1994, PASJ, 46, L147

Kotani, T., Kawai, N., Matsuoka, M., \& Brinkmann, W. 1996, PASJ, 48,619

Lamers, H. J. G. L. M., \& Cassinelli, J. P. 1999, Introduction to Stellar Winds (Cambridge: Cambridge University Press)

Lang, K. R. 1980, Astrophysical Formulae (Berlin: Springer-Verlag)

Larwood, J. 1998, MNRAS, 299, L32

Lipari, P. 1993, Astropart. Phys. 1, 195

Maccarone, J. T. 2002, MNRAS, 336, 1371

Mannheim, K., \& Schlickeiser, R. 1992, A\&A, 286, 983

Massi, M., Ribó, J. M., Paredes, J. M., et al. 2004, A\&A, 414, L1
Migliari, S., Fender, R., \& Méndez, M. 2002, Science, 297, 167319

Mioduszewski, A. J., Rupen, M. P., Hujellming, R. M., Pooley, G. G., \& Waltman, E. B. 2001, ApJ, 553, 766

Mirabel, I. F., \& Rodríguez, L. F. 1999, ARA\&A, 37, 409

Nolan, P. L, Tompkins, W. F., Grenier, I. A., \& Michelson, P. F. 2003, ApJ, 597, 615

Protheroe, R. J. 1998, ADP-AT-98-9 [arXiv: astro-ph/9812055]

Ogilvie, G. I., \& Dubus, G. 2001, MNRAS, 320, 485

Romero, G. E., Benaglia, P., \& Torres, D. F. 1999, A\&A, 348, 868

Romero, G. E., Kaufman-Bernadó, M. M., \& Mirabel, I. F. 2002, A\&A, 393, L61

Romero, G. E., \& Torres, D. F. 2003, ApJ, 586, L33

Romero, G. E., Torres, D. F., Kaufman-Bernadó, M. M., \& Mirabel, I. F. 2003, A\&A, 410, L1

Romero, G. E. 2004, Chin. J. A\&A, in press [arXiv: astro-ph/0407461]

Sarazin, C. L., Begelman, M. C., \& Hatchett, S. P. 1980, ApJ, 238, L129

Sierpowska, A., \& Bednarek, W. 2005, MNRAS, 356, 711

Stecker, F. W. 1979, ApJ, 228, 919

Torres, D. F., Romero, G. E., Combi, J. A., et al. 2001, A\&A, 370, 468

Torres, D. F., Domingo-Santamaría, E., \& Romero, G. E. 2004, ApJ, 601, L75

Vacca, W. D., Garmany, C. D., \& Shull, J. M. 1996, ApJ, 460, 914

Völk, H. J., \& Forman, M. 1982, ApJ, 253, 188

Volkova, L. V. 1980, Sov. J. Nucl. Phys. 31, 784

Weber, E. J., \& Davis, L. 1967, ApJ, 148, 217

White, R. L. 1985, ApJ, 289, 698

Wijers, R. A. M. J., \& Pringle, J. E. 1999, MNRAS, 308, 207 